\title{
Cultura de aprendizagem contínua, atitudes e desempenho no trabalho: uma comparação entre empresas do setor público e privado
}

Flávia de Souza Costa Neves Cavazotte

Pontifícia Universidade Católica do Rio de Janeiro / Escola de Negócios

Rio de Janeiro / RJ - Brasil

Valter de Assis Moreno Jr.

Faculdades IBMEC-RJ

Rio de Janeiro / RJ - Brasil

Lucas Martins Turano

Pontifícia Universidade Católica do Rio de Janeiro / Escola de Negócios

Rio de Janeiro / RJ - Brasil

Este artigo investiga a relação entre cultura de aprendizagem contínua, percepção de oportunidades de crescimento e desempenho individual, comparando empresas do setor público e privado. $\mathrm{O}$ trabalho se baseia em teorias que propõem que crenças compartilhadas sobre o apoio à aprendizagem no contexto organizacional são um importante fator situacional que pode influenciar as atitudes de funcionários e seu desempenho no trabalho. Foi realizada uma pesquisa de natureza quantitativa, por meio de questionários respondidos por funcionários da força de vendas de três organizações distintas, principais competidoras em um mesmo mercado, sendo duas delas empresas privadas e uma pública. Os resultados sugerem que a aprendizagem contínua está mais presente na cultura das empresas privadas, e está significativamente relacionada com a percepção de oportunidades de crescimento e com o desempenho.

Palavras-chave: cultura organizacional; gestão do conhecimento; aprendizagem contínua; administração pública; desempenho.

Cultura de aprendizaje contínua, actitudes y rendimento en el trabajo: una comparación de empresas en el sector público y privado

En este artículo se investiga la relación entre la cultura de aprendizaje continua, la percepción de oportunidades de crecimiento y el rendimiento individual, comparando las empresas en el sector público

DOI: http://dx.doi.org/10.1590/0034-7612136534

(c) (1)

Artigo submetido em 18 jun. 2014 e aceito em 15 maio 2015. 
y privado. El trabajo se lleva a las teorias que proponen que creencias compartidas sobre el apoyo al aprendizaje en el contexto de la organización son un importante factor situacional que puede influir en las actitudes de los empleados y su desempeño en el trabajo. Se realizó una encuesta cuantitativa mediante encuestas contestadas por los empleados de la fuerza de ventas de tres organizaciones distintas, principales competidores en el mismo mercado, dos de las cuales son privadas y una pública. Los resultados sugieren que el aprendizaje continuo es más presente en la cultura de las empresas privadas, y se relacionó significativamente con la percepción de las oportunidades de crecimiento y de rendimiento.

Palabras clave: cultura organizacional; gestión del conocimiento; aprendizaje continua; administración pública; performance.

Continuous learning culture, attitudes and job performance: comparing companies in public and private sectors

This article investigates the relationship among continuous learning culture, perception of growth opportunities and individual performance, comparing companies in the public and private sectors. The paper is based on theories that propose that shared beliefs about support for learning in the organizational context are an important situational factor that can influence the attitudes of employees and their job performance. A quantitative research was conducted with survey questionnaires answered by employees of the sales force of three distinct organizations, all major competitors in the same market, being two private and one public. The results suggest that continuous learning is more present in the culture of private companies, and it is significantly related to the perception of growth opportunities and performance.

KEYWORDs: organizational culture; knowledge management; continuous learning; public administration; performance.

\section{Introdução}

A cultura de uma organização é considerada um elemento crítico nas empresas, que influencia fortemente o comportamento de seus membros, as relações que estabelecem uns com os outros, a forma como tomam decisões e suas prioridades no trabalho (Kotter e Heskett, 2011). O tema tem sido objeto de estudo de um número crescente de trabalhos na literatura acadêmica brasileira (Silva e Fadul, 2010). Alguns pesquisadores têm sugerido que a cultura de uma organização pode estar associada à forma como o conhecimento é produzido e administrado nas empresas, o que constituiria um poderoso diferencial competitivo sustentável (Cardoso e Machado, 2008; Jackson et al., 2003). Nesse contexto, a chamada cultura de aprendizagem contínua (Miller, 1996; Tracey, Tannenbaum e Kavanagh, 1995) seria um elemento essencial nas estruturas de significado de organizações que se diferenciam pela capacidade de inovação, qualidade e competitividade. Esta pesquisa investiga variações na cultura de aprendizagem contínua em empresas com diferentes estruturas de capital, analisando também suas implicações sobre as atitudes e os comportamentos de seus funcionários, para assim melhor entender os problemas enraizados de eficiência e eficácia na gestão pública. 
Com a economia global em expansão e o aumento da competitividade, a capacidade de adaptar e ajustar rapidamente as operações de uma organização a novas condições pode representar a diferença entre prosperidade e crise em ambientes de mercado dinâmicos. Portanto, compreender as condições que facilitam a aprendizagem contínua e suas principais consequências sobre os trabalhadores teria não apenas implicações teóricas, mas, sobretudo, práticas (Sessa e London, 2006). No Brasil, embora diversos estudos tenham explorado a influência da cultura das organizações sobre aspectos como as políticas de gestão de pessoas e a gestão do conhecimento nas empresas (e.g., Santos, 2014; Souza et al., 2014), pesquisas com foco específico na aprendizagem contínua e suas consequências práticas ainda são raras.

Este trabalho contribui para a expansão desse campo do conhecimento e se baseia em dados levantados junto a funcionários da área comercial de três empresas do setor industrial, concorrentes no mesmo segmento de mercado, sendo duas privadas e uma pública. Os dados levantados nas empresas foram analisados por meio de equações estruturais, a fim de verificar as implicações do estímulo à aprendizagem contínua nas empresas sobre as atitudes de funcionários em relação às oportunidades na organização e seu desempenho formal e contextual.

Este artigo foi dividido em quatro seções, além da presente: o referencial teórico, onde os conceitos centrais em estudo são apresentados, bem como a literatura que discute esses temas na gestão pública, na qual as hipóteses do trabalho estão ancoradas. Em seguida, a metodologia utilizada é descrita, com ênfase no contexto da investigação e nos procedimentos de coleta de dados. A análise estatística, realizada com base na modelagem de equações estruturais, é apresentada em seguida, com as conclusões observadas para as hipóteses testadas neste trabalho; por fim, as implicações teóricas e práticas das observações da pesquisa de campo são discutidas na conclusão.

\section{Referencial teórico}

\subsection{Cultura organizacional}

A cultura é algo que está presente na vida das pessoas de forma dinâmica, sendo desempenhada e recriada continuamente a partir de interações sociais (Schein, 2009). Para Hofstede e Mccrae (2004:58), a cultura é definida como "uma programação mental coletiva que distingue um grupo ou uma categoria de pessoas, de outro". Apesar de suscitar uma ampla variedade de definições, há diversos elementos que são recorrentemente observados nas conceituações de cultura, como as noções de sistema informal, valores comuns e de coletividade. Nesse sentido, Schein (2009:16) explica que ela pode ser entendida como "um padrão de suposições básicas compartilhadas, que foi aprendido por um grupo à medida que solucionava seus problemas de adaptação externa e de integração interna".

Conforme destacado por Silva, Zanelli e Tolfo (2013), com base nos princípios da psicologia cognitiva e do interacionismo simbólico, podemos supor que o contexto psicossocial que denominamos cultura, formado pelo conjunto de pressupostos básicos comparti- 
lhados por um grupo social, é construído a partir das interações humanas e possui manifestações em diferentes níveis de análise. Os autores sugerem que em níveis mais profundos os pressupostos da cultura definem os limites entre o que é certo ou errado, o que é bom e o que é ruim. Em um nível mais consciente e acessível, os valores representariam aquilo que as pessoas desejam ou entendem ser o mais correto ou desejável em termos ideais, mas que nem sempre se coaduna com as suas práticas (Vasconcelos, Mascarenhas e Vasconcelos, 2004). Por fim, no nível mais tangível da cultura, observável em artefatos como documentos públicos e o contrato social e material estabelecido, ocorreria a intercepção da cultura com a percepção que as pessoas têm do ambiente psicossocial das organizações. A socialização dos indivíduos num contexto psicossocial particular teria, assim, um papel importante na construção dos padrões comportamentais peculiares a esse determinado grupo (Zanelli e Silva, 2008).

A função da cultura nas empresas seria servir como instrumento de reforço do sistema de valores vigente, diferenciando uma organização de outra qualquer (Pires e Macedo, 2006). Para Silva e Fadul (2010:4), “cultura organizacional deve abranger um conjunto de valores que refletem e se refletem em artefatos visíveis, que se alteram em função de fatores internos e/ou externos à própria cultura organizacional". Como pilar sustentador do presente estudo, a cultura organizacional é entendida a partir da definição de Pires e Macedo (2006), envolvendo inúmeros elementos e significados que são compartilhados entre membros de uma organização, de forma a nortear o sentir, pensar e agir desses indivíduos. Assim, a cultura teria o poder de influenciar de forma leve ou intensa, positiva ou negativamente, a forma como diferentes organizações lidam com o conhecimento e estimulam a aprendizagem.

No Brasil, os estudos observados na literatura têm procurado descrever a cultura das empresas e discutir algumas de suas implicações. Pesquisas recentes sugerem que a cultura organizacional tem consequências sobre a inovação (e.g., Faria e Fonseca, 2014; Morschel et al., 2013), sobre as políticas de gestão de pessoas praticadas nas empresas (e.g., Soares et al., 2012; Santos et al., 2014), sobre o grau de preocupação das organizações com sua responsabilidade social/ambiental (e.g., Rocha et al., 2011; Bianchi et al., 2013), sobre a liderança (e.g., Souza et al., 2004; Barreto et al., 2013) e sobre a própria gestão do conhecimento nas organizações (e.g., Souza et al., 2014; Angeloni e Grotto, 2009). Contudo, o foco em aprendizagem contínua na cultura de empresas públicas e privadas no Brasil, e suas consequências sobre as atitudes e comportamentos de funcionários, é um tema ainda não explorado nesse campo do conhecimento.

\subsection{Gestão do conhecimento}

Com o passar dos anos, o conhecimento vem sendo cada vez mais valorizado no ambiente organizacional (Massa e Testa, 2009), uma vez que ele seria uma base por meio da qual as organizações obteriam vantagens competitivas sustentáveis que as diferenciariam de ou- 
tras empresas (Cardoso e Machado, 2008). Entretanto, para muitas organizações a gestão do conhecimento apresenta ainda diversos desafios, já que ela envolve inúmeros e distintos elementos (Brito, Oliveira e Castro, 2012). No meio acadêmico, o interesse pela gestão do conhecimento aumentou bastante desde 1998 (Serenko e Bontis, 2004), apesar de alguns autores ainda considerarem esses estudos como limitados (Campos, 2007).

O conhecimento pode ser definido como uma combinação de "instintos, ideias, informações, regras e procedimentos que guiam ações e decisões" (Cardoso e Machado, 2008:5). Seguindo essa vertente, pode-se observar seu caráter complexo, mas essencial na tomada de quaisquer tipos de decisões, sugerindo a enorme importância de geri-lo efetivamente (Alavi, Kayworth e Leidner, 2006).

Partindo, também, da perspectiva de Cardoso e Machado (2008:6), a gestão do conhecimento pode ser conceituada como "o conjunto de atividades que busca desenvolver e controlar todo tipo de conhecimento em uma organização, visando à utilização na consecução de seus objetivos". Para Terra (2000), esse conjunto de atividades deve, primeiramente, englobar as demandas e características do ambiente, e ainda atentar-se para as necessidades, tanto individuais como coletivas, com o intuito de integrá-las nos processos de aprendizado de forma apropriada. Ainda segundo o autor, a gestão do conhecimento visa à aquisição, organização e comunicação, por parte dos empregados da organização, de dois tipos de conhecimentos que são propostos por Nonaka e Takeuchi (1997): tácito e explícito.

O conhecimento tácito é visto como aquele que é intrínseco ao indivíduo, ou seja, representa tudo aquilo que o indivíduo não expressa formalmente. Esse tipo está usualmente associado a competências, experiências, valores, emoções etc. Por outro lado, o conhecimento explícito é aquele capaz de ser expresso por vias tais que sua compreensão seja acessível a qualquer pessoa que entenda a simbologia empregada na transmissão desse conhecimento (Nonaka e Takeuchi, 1997). Entretanto, esse segundo tipo deve ser visto apenas como uma mínima parcela de todo o conhecimento existente (Nonaka, 1994). A partir disso, entende-se que as organizações, por meio da transformação de conhecimento tácito em explícito, captam, armazenam e transmitem todo esse novo conhecimento organizacional (Avelar, Vieira e Santos, 2011).

Essa interação, que pode ser considerada contínua, dos dois tipos de conhecimentos representa a chamada espiral de criação de todo o conhecimento, cujas etapas são: (1) socialização, (2) externalização, (3) internalização e (4) combinação (Nonaka e Takeuchi, 1997). A socialização estaria associada ao compartilhamento de experiências, permitindo, com isso, a conversão de conhecimento tácito em conhecimento tácito. Por sua vez, a externalização diz respeito à expressão de metáforas, conceitos, modelos e outros elementos visando ao encaminhamento do conhecimento tácito para o âmbito explícito (Cunha, Yokomizo e Capellini, 2011). A internalização, representada, por exemplo, pelo know-how, busca incorporar, individualmente, o conhecimento explícito em tácito. Por último, a combinação refere-se a um processo que converte conhecimento explícito em conhecimento explícito formando um amplo conceito, a partir da junção de dois ou mais conceitos formalizados, por parte dos próprios indivíduos. 


\subsection{Cultura organizacional e gestão do conhecimento na gestão pública}

Silva e Fadul (2010) buscaram analisar a produção científica brasileira sobre cultura organizacional em instituições públicas no período de 1997 a 2007, partindo da hipótese de que existiria uma vasta produção nessa temática. Contudo, nos 11 anos analisados, somente 47 artigos sobre o tema foram publicados no âmbito acadêmico, números considerados muito baixos pelos autores. Além disso, das 47 publicações, observou-se que somente $21,28 \%$ eram de base quantitativa e apenas 8,51\% eram estudos causais (Silva e Fadul, 2010).

Um dos trabalhos nesse universo, o artigo de Pires e Macedo (2006), investigou a cultura em organizações públicas brasileiras, constatando que o desenho desse tipo de organização é usualmente permeado por múltiplos níveis de hierarquia e complexos sistemas de organização. Os mesmos autores pontuaram o burocratismo, a interferência política advinda de fora da organização, o autoritarismo centralizado, o paternalismo, a aversão ao espírito empreendedor e a descontinuidade da gestão como características específicas inerentes às organizações públicas brasileiras.

Apesar da observação dessas características em uma vasta gama de empresas no setor público, no entender de Silva e Fadul (2007), desde 1995, a administração pública brasileira vem passando por mudanças significativas que visam promover uma maior eficiência na prestação dos serviços públicos. O objetivo do governo era estabelecer estruturas mais orgânicas, flexíveis e com uma maior celeridade, dando um caráter mais modernizado a essas instituições para atender às novas necessidades sociais. Por meio dessas iniciativas, a gestão pública estaria buscando minimizar os problemas anteriormente mencionados (Pires e Macedo, 2006).

Juntamente com os aspectos estruturais, aspectos relacionados à administração do conhecimento em organizações públicas são tidos como elementos essenciais para o entendimento e a análise de todo o processo de contínua geração de conhecimento e, consequentemente, para uma aprendizagem organizacional, sendo esse último o objeto específico de análise nesta pesquisa.

\subsection{Cultura de aprendizagem contínua}

Diversos autores discutem as características estruturais de aprendizagem no ambiente organizacional (Miller, 1996; Tracey, Tannenbaum e Kavanagh, 1995; Rosow e Zager, 1988; Dubin, 1990). Para Miller (1996), a aprendizagem organizacional é definida como a aquisição de conhecimento, por parte de indivíduos e grupos dispostos, visando sua aplicação na tomada de decisão e em atividades diversas, de maneira a influenciar as pessoas a realizar ações consideradas importantes para a organização. Nesse contexto, encontra-se a noção de aprendizagem organizacional contínua.

Sob a ótica de Tracey e colaboradores (1995), culturas de aprendizagem contínua são aquelas caracterizadas por percepções compartilhadas de que o aprendizado é central nas ati- 
vidades e em todas as dimensões laborais. Schein (2009) complementa essa ideia, ao propor que organizações com uma forte cultura de aprendizagem contínua trariam esse aprendizado incorporado ao trabalho de todos e nas estruturas de significado em curso na organização. Enquanto a simples aprendizagem organizacional remete a eventos triviais e temporários, a aprendizagem continuada envolveria aspectos muito mais complexos e, portanto, de difícil reprodução. Como resultado, a aprendizagem contínua se constitui em uma poderosa fonte de vantagem competitiva sustentável (Jackson, Hitt e Denisi, 2003).

A despeito de ser um importante elemento na gestão, o foco em aprendizagem contínua parece ser criado a partir de certas características organizacionais, e seria favorecido por elementos intrínsecos à cultura das organizações (Tracey et al., 1995). A prática da administração do conhecimento nas empresas públicas tem sido analisada por diversos autores, principalmente no que se refere aos entraves que ainda se imporiam a seu pleno desenvolvimento e que dificultam sua eficácia. Como grande parte do conhecimento gerado acaba sendo armazenado somente na mente das pessoas, a rotatividade, as aposentadorias e transferências de pessoal acarretariam a perda de know-how e capital intelectual.

Ademais, na visão de Brito, Oliveira e Castro (2012), instituições públicas ainda permaneceriam com características estruturais da administração clássica, e, nelas, as experiências, julgamentos e rotinas desenvolvidas no contexto laboral ainda não seriam tratadas como elementos geradores de novos conhecimentos. Os autores também sugerem que nas organizações públicas o conhecimento ainda é visto de maneira uniformizada, como comportamentos regulares e previsíveis. Por essa razão, elementos estruturais e culturais em empresas privadas favoreceriam muito mais a aprendizagem contínua, enquanto a ausência desses mesmos elementos, e os prejuízos causados pelas descontinuidades e interferências políticas, burocratismos e estruturas centralizadas de autoridade, representariam barreiras à criação de uma cultura com foco em aprendizagem contínua no segmento público. Assim, neste trabalho apresentamos a seguinte proposição:

Hipótese 1: Empresas privadas tendem a ter culturas que reforçam mais a aprendizagem contínua do que empresas públicas.

De acordo com Alavi e Leidner (1999), em organizações sem uma estratégia explícita para melhor criar e integrar o conhecimento, os sistemas existentes para facilitar a comunicação e compartilhamento de informação têm apenas um efeito marginal. Da mesma forma, Markus (2001) argumenta que devem existir incentivos adequados para que os produtores de conhecimento passem a articular e registrar suas ideias de maneira que possam ser úteis aos outros. Assim, a eficácia e o sucesso em longo prazo de tais práticas dependem não só de tecnologias, mas também da resolução eficaz das questões culturais e organizacionais (Alavi et al., 2006).

Nesse contexto, por terem o conhecimento e o aprendizado como fatores essenciais que permeiam todos os níveis e áreas da empresa (Tracey et al., 1995), empresas com forte cultura de aprendizagem contínua se destacariam por serem detentoras de sistemas que oferecem mais oportunidades para desenvolvimento profissional e pessoal, e a importância 
do desempenho diferenciado de seu pessoal (Dubin, 1990). Assim, o foco em aprendizagem contínua promoveria a valorização da aprendizagem como elemento fundamental para todas as dimensões e atividades de trabalho.

Ambientes de trabalho permeados pela cultura de aprendizagem contínua possuem características que claramente denotam a ênfase no conhecimento, como valores associados à inovação, qualidade e competitividade, e políticas voltadas para o desenvolvimento de funcionários (Tracey et al., 1995). O foco em aprendizagem não seria limitado apenas ao treinamento formal, mas também incluiria mecanismos informais, como as trocas entre supervisores e equipes, e também estruturas que estimulam esforços progressivos e inovadores.

Uma vez que, do ponto de vista individual, o desenvolvimento contínuo de habilidades e competências é também uma necessidade, e proporciona aos trabalhadores o senso de segurança e empregabilidade (Cuyper et al., 2008), pode-se esperar que indivíduos imersos em organizações com forte cultura de aprendizagem contínua tenham maiores oportunidades para desenvolvimento pessoal e profissional. Em outras palavras, quanto mais forte a cultura de aprendizagem contínua e seus elementos estruturais em uma organização, tais como investimentos diretos nos funcionários pela empresa e suas lideranças, maiores as oportunidades de adquirir experiências novas no trabalho e o senso de valor agregado à formação pessoal por meio da organização (Lee e Bruvold, 2003). Dessa forma, a percepção de oportunidades de crescimento potencial nessas empresas seria maior, em decorrência dos investimentos e incentivos inerentes à cultura de aprendizagem contínua. Assim, neste trabalho apresentamos a seguinte proposição:

Hipótese 2: Indivíduos que trabalham em organizações detentoras de uma forte cultura para aprendizagem contínua percebem mais oportunidades de crescimento na empresa.

\subsection{Implicações para o desempenho}

Muito embora o desempenho possa ser estudado em diferentes níveis de análise, como o desempenho das organizações ou de equipes de trabalho (Bastos et al., 2004), este artigo discute especificamente o desempenho individual. O desempenho individual no trabalho é aqui compreendido como um conjunto de comportamentos apresentados pelos indivíduos em um contexto específico, e é determinado por aquilo que tais indivíduos acreditam ser esperado deles nessa situação (Bateson, 1972; Bendassolli e Malvezzi, 2013).

Segundo Bendassolli e Malvezzi (2013), sendo o desempenho um fenômeno complexo, diferentes perspectivas teóricas se somam para discutir seus múltiplos determinantes no nível individual, grupal e contextual. Particularmente relevante para este estudo, o modelo dos inibidores e facilitadores do desempenho (Waldman e Avolio, 1993) aponta para a importância de fatores situacionais associados ao sistema organizacional na compreensão do desempenho. De acordo com essa perspectiva, aspectos intrínsecos ao ambiente de trabalho, como a disponibilidade de recursos e a cultura organizacional, teriam impacto tanto sobre aspectos pro- 
cessuais do desempenho, ou seja, os comportamentos individuais, como sobre os resultados alcançados no trabalho e suas consequências.

Na literatura sobre desempenho, os pesquisadores têm feito uma distinção entre o desempenho formal nas tarefas específicas de trabalho e o desempenho individual contextual. O desempenho formal, ou na tarefa, é definido como a capacidade do indivíduo de realizar o papel que lhe é formalmente atribuído pela organização (Borman e Motowidlo, 1993), papel este que está normalmente descrito na estrutura de cargos de uma empresa. O desempenho formal está associado ao grau de proficiência com que um indivíduo desempenha ações definidas formalmente nas organizações, e que, por serem relevantes para a geração de seus produtos ou serviços, têm também implicações para o alcance das metas organizacionais (Campbell et al., 1993). Além disso, o desempenho nesses papéis é também um elemento importante do contrato psicológico estabelecido entre funcionários e empresa (Rousseau, 1995), costuma ser mensurado em termos de níveis ou margens de contribuição, e está associado, em maior ou menor grau, às recompensas diretas e indiretas recebidas pelos indivíduos no trabalho, que estão estabelecidas nos sistemas de gestão do desempenho (Schiemann, 2009).

Em contrapartida, o desempenho contextual é descrito como um conjunto de comportamentos espontâneos, discricionários (Rego e Cunha, 2008), porém também importantes para o bom funcionamento da organização, e que vão além do esperado nos papéis formais. Atualmente, a maior parte dos pesquisadores se refere ao desempenho contextual como comportamentos de cidadania organizacional (Organ, Podsakoff, e MacKenzie, 2006), conceito enfatizado em estudos sobre desempenho no trabalho principalmente a partir da década de 1980 (Podsakoff et al., 2009). No Brasil, Siqueira (2003) e Siqueira e Gomide (2004) contribuíram para ampliar a discussão sobre cidadania organizacional, e definem esses comportamentos como ações espontâneas que não estão previstas nos contratos formais de trabalho, que emergem a partir das trocas sociais e beneficiam os sistemas organizacionais (Bastos, Siqueira e Gomes, 2014). Em suas pesquisas, esses autores têm observado a influência de fatores psicossociais associados ao ambiente de trabalho na cidadania organizacional, com ênfase no grau de suporte oferecido aos funcionários pela organização e na justiça organizacional.

A presença de mais oportunidades de crescimento pode afetar o desempenho dos colaboradores, mesmo que indiretamente. Tanto interna quanto externamente às organizações, o ambiente de aprendizagem contínua é caracterizado como detentor, essencialmente, de inovação e competição (Rosow e Zager, 1988). Segundo Tracey e colaboradores (1995), em empresas com culturas de aprendizagem contínua, uma série de sistemas é posta em prática de modo a facilitar a aquisição e transferência de conhecimento, e os funcionários são estimulados a exceder em suas áreas de responsabilidade. Toda a organização trabalha ativamente, a fim de ser uma das melhores na sua indústria/mercado. Assim, nesses contextos haveria uma forte expectativa de que todos os membros que estão na organização tenham um alto nível de desempenho laboral, visando o progresso por meio do esforço máximo. 
A presença de oportunidades de crescimento funciona também como um estímulo adicional ao bom desempenho nas atribuições de trabalho por parte dos funcionários. Isso se dá tanto por conta da valorização do crescimento na carreira e de desenvolvimento no trabalho pelos indivíduos na atualidade (e.g., Economist Intelligence Unit, 2010), como em função da escassez de ofertas de tais oportunidades nas empresas de hoje (Dutra, 2010). Uma vez que essas oportunidades também têm um importante papel nos sistemas de recompensa e reconhecimento pelo trabalho bem-feito (e.g., Kuvaas e Dysvik, 2009), neste trabalho apresentamos as seguintes proposições:

Hipótese 3a: Indivíduos que trabalham em organizações detentoras de uma forte cultura para aprendizagem contínua apresentam maior nível de desempenho formal no trabalho.

Hipótese 3b: Indivíduos que acreditam ter mais oportunidades de crescimento apresentam maior nível de desempenho formal no trabalho.

Por serem ambientes nos quais sistemas formais e informais estimulam a produção, armazenagem e transferência de conhecimentos, as organizações com fortes culturas de aprendizagem contínua não somente estimulariam, mas teriam no compartilhamento de conhecimentos e na cooperação interpessoal e entre equipes algumas de suas características constitutivas (Cunha, Yokomizo e Capellini, 2011). Nesses ambientes, processos e sistemas que permitem agregar conhecimentos por intermédio de conversas a distância, armazenagem e troca de documentos formais ou redes de comunicação internas, não só existem, mas seriam utilizados efetivamente por seus membros (Moreno e Oliveira, 2010), uma vez que a cultura de aprendizagem contínua que permeia a empresa incentiva e justifica seu uso. Assim, as culturas de aprendizagem seriam celeiro não apenas de uma gestão do conhecimento mais eficaz, mas também facilitariam diversos comportamentos tipicamente considerados dentro do escopo da cidadania organizacional, tais como ajudar colegas no trabalho, tomar iniciativas para aprimorar produtos ou serviços e gerar inovações, defender os interesses da organização e buscar seu autodesenvolvimento, entre outros (Podsakoff, Mackenzie, Paine e Bachrach, 2000).

A satisfação com as oportunidades de crescimento no trabalho parece estar associada aos comportamentos de ajuda, com a dedicação à organização e a defesa entusiasmada da empresa (Jawahar, 2012). Assim, a perspectiva de crescimento favoreceria todo um conjunto de atitudes positivas do funcionário diante da organização, como a percepção de que a empresa premia seus funcionários de maneira justa, a satisfação pessoal no trabalho, a motivação para se envolver nas tarefas trabalho e o senso de comprometimento com a organização; todas essas atitudes que têm sido sistematicamente associadas à cidadania organizacional em pesquisas já realizadas (Organ et al., 2006; Podsakoff et al., 2009). Dessa forma, neste trabalho apresentamos as seguintes proposições:

Hipótese 4a: Indivíduos que trabalham em organizações detentoras de uma forte cultura para aprendizagem contínua apresentam maior cidadania organizacional.

Hipótese 4b: Indivíduos que acreditam ter mais oportunidades de crescimento apresentam maior cidadania organizacional.

As ideias discutidas e as hipóteses a serem testadas no presente estudo estão resumidas no modelo seguinte. 
Figura 1

Modelo proposto

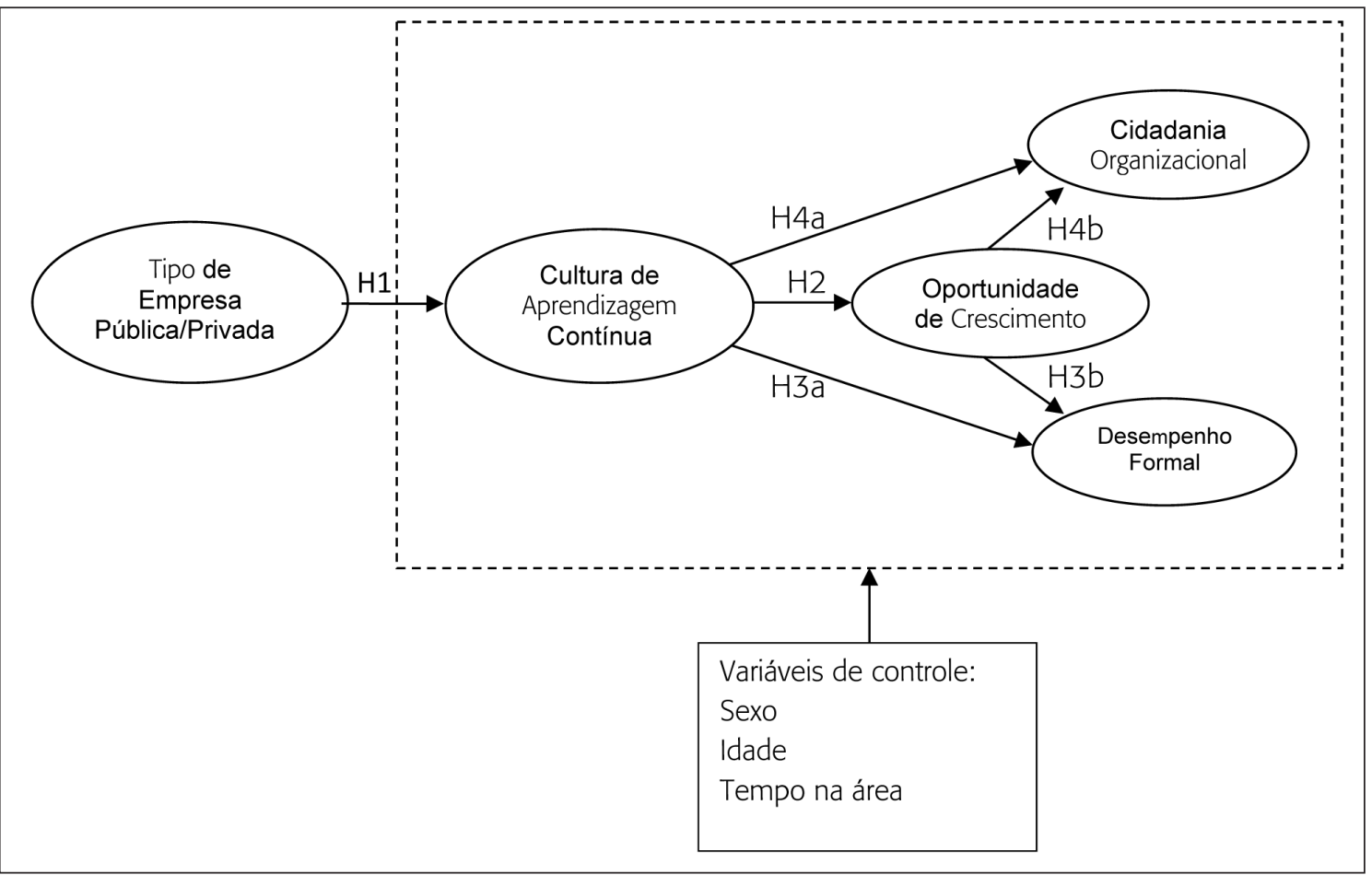

Fonte: Elaborada pelos autores.

\section{Método de pesquisa}

As hipóteses apresentadas na seção anterior foram verificadas nesta pesquisa por meio de dados levantados em três organizações que atuam e são concorrentes em um mesmo mercado e comercializam os mesmos tipos de produtos, sendo duas pertencentes ao setor privado e uma pertencente ao setor público. Essas organizações são similares em termos de estrutura organizacional, embora variem em sua participação em outros segmentos da cadeia produtiva em que atuam. De forma geral, elas representam três das quatro empresas com maior percentual de participação de mercado nesse segmento, com pequenas variações entre elas. Por força de compromisso de confidencialidade assumido com as organizações em questão, tanto o segmento específico como os nomes das empresas foram suprimidos, com o intuito de preservar seu anonimato. Neste artigo, as organizações privadas são identificadas como empresa Alfa e Beta, e a organização pública como empresa Gama.

Os dados foram levantados por meio de amostragem por critério de conveniência, uma vez que os funcionários foram convidados a participar voluntariamente da pesquisa por oca- 
sião da prestação de serviços consultivos nessas organizações. Todas as empresas se localizam no Rio de Janeiro, o que facilitou também o acesso aos participantes, que são funcionários da força de vendas das três organizações e têm atribuições similares. Os dados foram levantados por meio do mesmo procedimento nas três empresas. Os questionários foram distribuídos aos participantes pelo consultor em mãos, e lhes foi garantido total anonimato. Os questionários foram depositados em um malote único pelos participantes em cada empresa, que foi recolhido ao final da prestação de serviços.

Para a coleta de dados, adotou-se um questionário pré-estruturado, composto por duas partes. Na primeira, foram feitas perguntas sociodemográficas e na segunda parte foram feitas perguntas sobre o desempenho, a cidadania organizacional, percepção de oportunidades de crescimento e cultura de aprendizagem contínua. Para avaliar a cidadania organizacional, foi utilizada a escala criada por Gomide Jr. (1999), com foco no comportamento extrapapel, cujo alpha de Cronbach original é 0,88. São exemplos de itens dessa escala: "realizo trabalhos além das atribuições de meu cargo" e "apresento espontaneamente sugestões de melhoria na organização". As perguntas referentes à percepção de oportunidades de crescimento têm como base o modelo de Hackman e Oldham (1980), traduzido e adaptado por Moraes e Kilimnik (1994). Foi utilizada nesta pesquisa a subescala sobre a possibilidade de crescimento, com pequenas alterações para ressaltar as oportunidades existentes. São exemplos os itens "tenho oportunidades de crescimento pessoal e desenvolvimento no trabalho" e "tenho oportunidade de enfrentar desafios no trabalho". Por último, as questões que buscam medir a cultura para aprendizagem foram baseadas na escala desenvolvida por Tracey, Tannenbaum e Kavanagh (1995), cujos itens se referem ao padrão compartilhado de significados e expectativas sobre as condições facilitadoras e restritivas à aprendizagem nas organizações (Abbad et al., 2006). No Brasil, Pantoja e Freitas (2003) construíram a escala de suporte à aprendizagem contínua com base nesse modelo, com alpha de Cronbach original igual a 0,96. São exemplos de itens utilizados neste trabalho: "nesta empresa, as pessoas são estimuladas a buscar novas informações e conhecimentos sobre o trabalho" e "nesta empresa, as pessoas são estimuladas a compartilhar novos conhecimentos".

Duas das empresas participantes não autorizaram a divulgação de métricas internas de desempenho formal (desempenho em vendas), nem o envolvimento de terceiros (ex: supervisores) como avaliadores, o que inviabilizou o uso adequado de escalas de desempenho disponíveis na literatura. Assim, foi adotado nesta pesquisa o construto importância do desempenho formal, que reflete a percepção dos participantes sobre as implicações de sua produtividade para a carreira e continuidade no cargo e na empresa, ou seja, as consequências ou efeitos individuais do desempenho (Peixoto e Caetano, 2013). O construto foi mensurado com três itens elaborados pelos autores, que solicitavam ao participante que avaliasse, numa escala de um a cinco, o nível de importância de sua produtividade para fins de promoção, substituição, ou demissão do seu cargo atual na empresa. Para a mensuração dos demais construtos, foram empregadas escalas Likert de cinco pontos, com alternativas de resposta variando entre um (discordo completamente) e cinco (concordo completamente). A exceção foi a variável dummy Tipo de Empresa, que assume o valor 0, quando a empresa do respondente é privada, e 1, quando a empresa é pública. 
Foram incluídas na análise três variáveis demográficas de controle: idade, sexo e tempo na área. Variáveis demográficas são habitualmente selecionadas para esse fim, dado o seu potencial efeito nas percepções e experiências dos indivíduos, como é o caso dos construtos avaliados nesta pesquisa (Atinc, Simmering e Kroll, 2011; Spector e Brannick, 2010). As três variáveis de controle utilizadas neste estudo foram inseridas em todas as avaliações das relações causais previstas no modelo proposto.

As hipóteses propostas no artigo foram avaliadas com técnicas estatísticas de equações estruturais baseadas em Partial Least Squares (PLS). Diferentemente do método de equações estruturais baseado em covariâncias (Structural Equation Modeling - SEM), o PLS utiliza os dados coletados para estimar os efeitos propostos que maximizem a variância total explicada na amostra. Entre as vantagens do método em relação ao SEM, destacam-se a possibilidade de se trabalhar com amostras menores do que as tipicamente exigidas no método baseado em covariância, e a robustez em relação a violações da premissa de normalidade multivariada, à multicolinearidade entre e em blocos de variáveis manifestas, e a problemas de especificação do modelo de equações estruturais (Chin, 2010). Para realizar análises, empregou-se o software SmartPLS v. 2.0.M3 (Ringle, Wende e Will, 2014).

\section{Análise de resultados}

Foram recebidos 165 questionários dos participantes das três empresas contempladas na pesquisa. Destes, 131 estavam completos e puderam ser aproveitados nas análises. A amostra coletada foi composta por 71 participantes da empresa Gama, 34 da empresa Alfa e 26 da empresa Beta. Conforme exposto na tabela 1 , nas três empresas, o percentual de respondentes do sexo masculino foi bem superior ao de respondentes do sexo feminino; no entanto, este tende a ser o perfil demográfico típico neste segmento ocupacional. Quanto ao nível educacional, observa-se que as empresas Gama e Beta apresentam um perfil similar, com maior concentração de respondentes com nível superior completo e nível de especialização, enquanto na empresa Alfa há maior concentração nos níveis de especialização e de mestrado/doutorado.

Tabela 1

Frequências obtidas na amostra

\begin{tabular}{|lcccccc|}
\hline \multirow{2}{*}{ Empresa } & \multicolumn{2}{c}{ Sexo (\%) } & & \multicolumn{3}{c|}{ Nível educacional (\%) } \\
\cline { 2 - 3 } \cline { 5 - 7 } & Masculino & Feminino & & $\begin{array}{c}\text { Superior } \\
\text { Completo }\end{array}$ & $\begin{array}{c}\text { Especialização } \\
\text { Completa }\end{array}$ & $\begin{array}{c}\text { Mestrado } \\
\text { Completo }\end{array}$ \\
\hline Alfa & 79,4 & 20,6 & & 11,8 & 50,0 & 38,2 \\
Beta & 84,6 & 15,4 & & 57,7 & 34,6 & 7,7 \\
Gama & 90,1 & 9,9 & & 57,7 & 35,2 & 7,0 \\
Total & 86,3 & 13,7 & & 45,8 & 38,9 & 15,3 \\
\hline
\end{tabular}

Fonte: Elaborada pelos autores. 
A tabela 2 descreve as estatísticas descritivas básicas para as demais variáveis demográficas coletadas no estudo. Em geral, observa-se uma considerável similaridade entre os valores de média e desvio-padrão observados para as variáveis idade e tempo de experiência, nas três empresas. Por outro lado, os tempos médios no setor e na empresa parecem ser bem menores na empresa Alfa do que nas outras duas.

Tabela 2

Estatísticas descritivas

\begin{tabular}{|c|c|c|c|c|c|c|c|c|}
\hline \multirow[b]{2}{*}{ Empresa } & \multicolumn{2}{|c|}{$\begin{array}{l}\text { Idade } \\
\text { (anos) }\end{array}$} & \multicolumn{2}{|c|}{$\begin{array}{l}\text { Tempo no setor } \\
\text { (anos) }\end{array}$} & \multicolumn{2}{|c|}{$\begin{array}{c}\text { Tempo na empresa } \\
\text { (anos) }\end{array}$} & \multicolumn{2}{|c|}{$\begin{array}{c}\text { Tempo de experiência } \\
\text { (anos) }\end{array}$} \\
\hline & Média & $\begin{array}{l}\text { Desvio } \\
\text { Padrão }\end{array}$ & Média & $\begin{array}{l}\text { Desvio } \\
\text { Padrão }\end{array}$ & Média & $\begin{array}{l}\text { Desvio } \\
\text { Padrão }\end{array}$ & Média & $\begin{array}{l}\text { Desvio } \\
\text { Padrão }\end{array}$ \\
\hline Alfa & 42,9 & 8,6 & 3,8 & 2,8 & 7,1 & 5,7 & 17,9 & 10,6 \\
\hline Beta & 43,7 & 9,8 & 5,5 & 3,4 & 10,7 & 7,8 & 18,4 & 10,4 \\
\hline Gama & 42,9 & 9,2 & 8,0 & 4,9 & 11,6 & 7,2 & 17,5 & 9,0 \\
\hline Total & 43,1 & 9,1 & 6,4 & 4,5 & 10,2 & 7,2 & 17,8 & 9,7 \\
\hline
\end{tabular}

Fonte: Elaborada pelos autores.

Para avaliar o modelo de mensuração empregado no teste das hipóteses de pesquisa, foi realizada primeiramente uma análise fatorial confirmatória (Confirmatory Factor Analysis - CFA), de acordo com o método delineado por Wetzels, Odekerken-Schröder e van Oppen (2009). Os resultados obtidos foram avaliados quanto a sua aderência aos critérios de validade convergente e discriminante descritos por Chin (2010), a saber: (1) cargas altas e significantes das variáveis observadas em suas respectivas variáveis latentes, e baixas e/ ou não significantes nas demais variáveis do modelo; (2) pouca variação entre as cargas dos indicadores de uma variável latente; (3) valores de variância média extraída (Average Variance Extracted - AVE) iguais ou superiores a 0,50; (5) valores de confiabilidade composta (Composite Reliability - CR) iguais ou superiores a 0,70; e (6) a raiz quadrada de AVE para uma variável latente maior que as correlações dessa variável com as demais. Além disso, recomenda-se que os valores de alfa de Cronbach para as variáveis latentes sejam iguais ou superiores a 0,70 (Hair, Black e Babin, 2009). Para que os critérios fossem plenamente atendidos, eliminaram-se, num processo iterativo, alguns indicadores das escalas originais. Os resultados das análises confirmatórias subsequentes indicaram que o modelo de mensuração do estudo apresentava validades convergente e discriminante adequadas, ratificando as qualidades psicométricas das escalas nesta amostra. A tabela 3 sintetiza os achados finais dessa etapa da análise de dados, e apresenta os valores observados de CR, AVE e alfa de Cronbach $\left(\alpha_{C}\right)$ das escalas. 
Tabela 3

Resultados obtidos para o modelo de mensuração

\begin{tabular}{|lccccccc|}
\hline \multicolumn{1}{|c}{ Variáveis Latentes } & Cargas & Médiaa $^{\text {a }}$ & $\begin{array}{c}\text { Desv. } \\
\text { Padrão }^{\text {a }}\end{array}$ & CR & AVE & $\begin{array}{c}\text { Correlação } \\
\text { máxima }^{\text {b }}\end{array}$ \\
\hline Cultura de aprendizagem contínua (CA) & $0,70-0,84$ & 4,15 & 1,25 & 0,87 & 0,63 & 0,80 & 0,77 \\
Desempenho Formal (DES) & $0,81-0,87$ & 2,29 & 0,96 & 0,89 & 0,73 & 0,82 & 0,74 \\
Oportunidades de crescimento (OC) & $0,86-0,92$ & 3,60 & 1,24 & 0,88 & 0,71 & 0,80 & 0,77 \\
Cidadania organizacional (CCO) & $0,93-0,94$ & 3,46 & 1,09 & 0,90 & 0,57 & 0,87 & 0,77 \\
\hline
\end{tabular}

Fonte: Elaborada pelos autores.

a Média e desvio padrão foram calculados com base nos escores gerados pelo PLS para as variáveis latentes.

b Maior correlação (em valor absoluto) com as demais variáveis latentes.

Em análises de equações estruturais baseadas em PLS, a qualidade do modelo estrutural é primariamente avaliada em função da proporção de variância explicada $\left(\mathrm{R}^{2}\right)$ para as variáveis latentes endógenas incluídas no estudo (Urbach e Ahlemann, 2010). Adicionalmente, a significância estatística dos coeficientes estimados deve ser determinada por meio de técnicas de reamostragem, tais como bootstrapping e blindfolding (Chin, 2010). No presente estudo, adotou-se a técnica de bootstrapping, com o tamanho de amostra igual a 131 (o tamanho da amostra coletada), e o número de reamostragens igual a 500. A figura 2 apresenta os resultados obtidos para os efeitos previstos nas hipóteses anteriormente descritas.

\section{Figura 2}

\section{Resultados para o modelo estrutural}

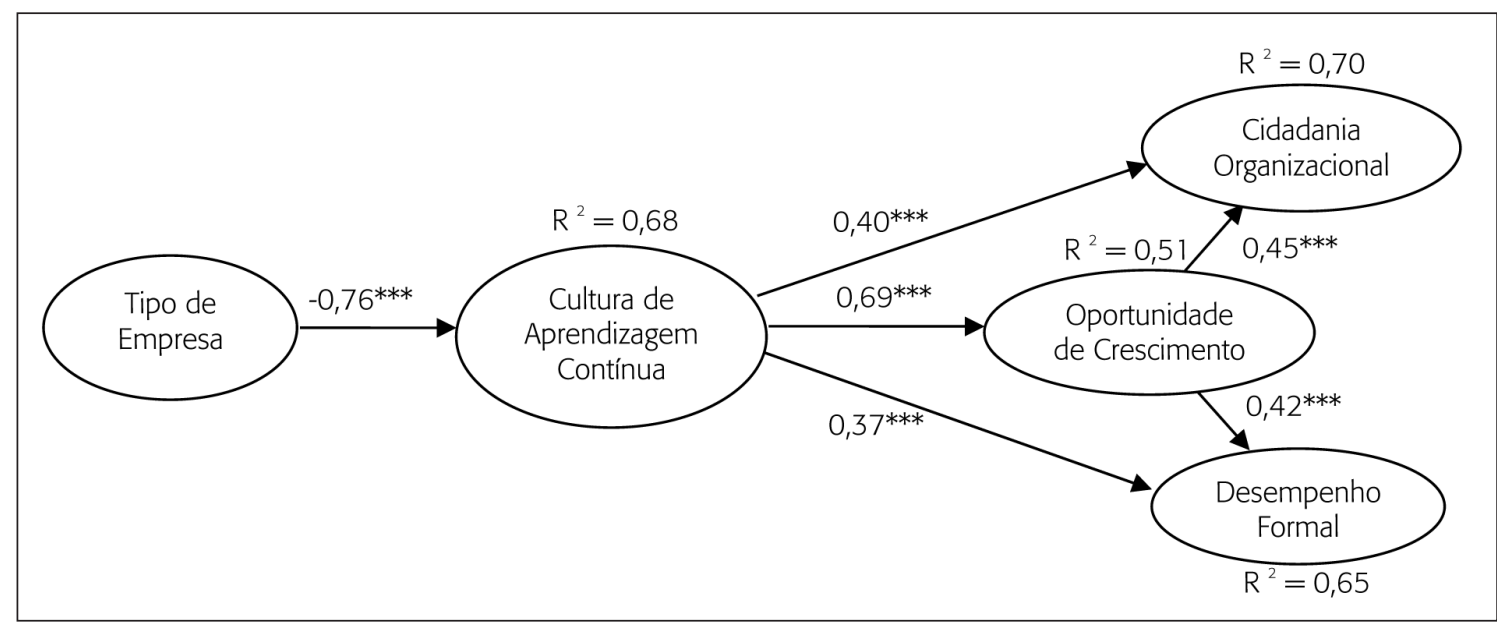

Fonte: Elaborada pelos autores.

Nota: *** $\mathrm{p}<0,001$; Tipo de Empresa: "0" = privada; "1" = pública. 
O modelo foi capaz de explicar proporções elevadas da variância das variáveis latentes endógenas, apresentando um $\mathrm{R}^{2}$ médio de 0,64, com valores individuais entre 0,51 e 0,70. Dessa forma, considera-se que sua capacidade preditiva é satisfatória, na medida em que reproduz adequadamente as variações observadas na amostra.

Os resultados proveem suporte empírico para todas as hipóteses delineadas no estudo. Conforme esperado, a variável dummy Tipo de Empresa, que indicava se o respondente trabalhava numa empresa pública (valor $=1$ ) ou privada (valor $=0$ ), teve um efeito negativo e estatisticamente significante na percepção do indivíduo da existência de uma cultura de aprendizado em sua organização $(\beta=-0,76$; $p<0,001)$. Confirma-se, portanto, a proposição de que empresas públicas tendem a apresentar, na percepção de seus funcionários, culturas menos voltadas para o aprendizado (H1).

Por sua vez, a percepção do funcionário da existência de uma cultura de aprendizado em sua empresa parece ter uma influência direta e positiva em seu comportamento de cidadania corporativa, em sua percepção da existência de oportunidades de crescimento em seu emprego, e em seu desempenho formal. Os respectivos efeitos estimados foram todos positivos e estatisticamente significantes $\left(\beta_{\mathrm{CA}-\mathrm{OCB}}=0,40 ; \beta_{\mathrm{CA}-\mathrm{OC}}=0,69 ; \beta_{\mathrm{CA}-\mathrm{DES}}=0,37 ; \mathrm{p}<0,001\right)$, destacando-se entre eles o de cultura de aprendizagem contínua em cidadania organizacional, que foi substancialmente mais forte do que os outros dois. Por conseguinte, conclui-se que as hipóteses H1, H2, H3a e H4a obtiveram suporte empírico no estudo.

A percepção de oportunidade de crescimento também apresentou efeitos diretos positivos e estatisticamente significantes nas variáveis cidadania organizacional e desempenho formal $\left(\beta_{\text {OC-OCB }}=0,45 ; \beta_{\text {OC-DES }}=0,42 ; \mathrm{p}<0,001\right)$. Confirmam-se, assim, as hipóteses H3b e H4b propostas no estudo. Tendo em vista os resultados descritos, pode-se ainda afirmar que a percepção de oportunidades de crescimento atua como um mediador parcial dos efeitos das culturas de aprendizagem contínua na cidadania organizacional e no desempenho formal. De fato, os testes dos respectivos efeitos compostos foram ambos estatisticamente significantes $\left(\beta_{\text {CA-OC-OCB }}=0,32 ; \beta_{\text {CA-OC-DES }}=0,28 ; \mathrm{p}<0,001\right)$. Além disso, os efeitos compostos do tipo de empresa nas variáveis oportunidade de crescimento, cidadania organizacional e desempenho foram todos negativos e estatisticamente significantes $\left(\beta_{\text {TE-CA-OC }}=-0,52 ; \beta_{\text {TE-CA-OC-OCB }}=-0,54\right.$; $\left.\beta_{\text {TE-CA-OC-DES }}=-0,50 ; \mathrm{p}<0,001\right)$. Isso indica que funcionários da empresa pública tendem a perceber menos oportunidades de crescimento em seus ambientes de trabalho e, por conseguinte, a terem níveis mais baixos de cidadania organizacional e desempenho do que seus pares em empresas privadas.

Por fim, observou-se que, das variáveis de controle, apenas o tempo no setor teve efeitos estatisticamente significantes. Os resultados sugerem que essa variável tende a afetar negativamente tanto a percepção do indivíduo da existência de uma cultura voltada para o aprendizado em sua empresa $(\beta=-0,17$; $<<0,05)$, quanto o desempenho formal $(\beta=-0,19 ; \mathrm{p}<0,01)$. 


\section{Discussão e conclusão}

Este estudo teve como objetivo analisar o grau de ênfase em aprendizagem contínua percebido na cultura de empresas e sua associação com oportunidades de crescimento, cidadania organizacional e desempenho formal, comparando as percepções, atitudes e comportamentos de funcionários em empresas públicas e privadas, concorrentes no mesmo mercado. As análises realizadas indicaram que o foco em aprendizagem contínua variou significativamente na percepção dos funcionários da área comercial das empresas estudadas, sendo mais forte nas empresas privadas do que na pública.

Por meio da análise dos dados deste estudo, também foi possível observar a associação positiva entre a ênfase em aprendizagem contínua na cultura das empresas e as atitudes dos funcionários em relação às oportunidades de crescimento nas organizações, sendo maior nas empresas onde há maior ênfase em aprendizagem. Além disso, a força da aprendizagem contínua na cultura das empresas também está positivamente associada a uma maior cidadania organizacional e importância do desempenho formal nos grupos estudados por via da percepção de oportunidade de crescimento. Tanto a importância do desempenho formal como o contextual foram maiores na medida em que os funcionários acreditam que há mais oportunidade de crescimento nas empresas.

O presente trabalho representa uma importante contribuição para o avanço do conhecimento sobre aprendizagem contínua no contexto empresarial. Algumas ideias anteriormente expostas por Dubin (1990) e Schein (2009) foram confirmadas - em ambientes onde a aprendizagem contínua é um forte elemento na cultura organizacional, mais oportunidades de desenvolvimento também parecem ser promovidas, e o desempenho e engajamento no trabalho tendem a ser maiores. Além disso, o estudo confirma as premissas de Brito, Oliveira e Castro (2012), ao observar que funcionários das empresas privadas tenderam a indicar uma cultura de aprendizagem contínua mais forte em suas empresas do que os da empresa pública. Uma vez que esses funcionários têm as mesmas atribuições e atuam em empresas concorrentes num mesmo setor, pode-se afirmar que as diferenças observadas parecem estar fortemente associadas a características intrínsecas às organizações em estudo. Sendo essa a configuração em um setor onde há concorrência entre empresas, o que notadamente estimula a produtividade e eficiência organizacional, é possível especular que a aprendizagem contínua seja ainda menos enfatizada em empresas públicas que são provedoras exclusivas de serviços.

Essas observações têm diversas implicações práticas. Na atualidade, organizações que conseguem constantemente aprender e se aperfeiçoar adquirem uma fonte profusa de diferencial competitivo baseado em conhecimento (Jackson et al., 2003), além de estimular intelectualmente e reter seu corpo funcional, o que também contribui para o fortalecimento do capital humano dessas empresas (Ployhart e Moliterno, 2011). Assim, em condições de igualdade de concorrência, não seria surpresa que empresas privadas conquistassem fatias ainda maiores de seus mercados, a persistirem as diferenças observadas neste estudo. Além disso, o foco na aprendizagem contínua também costuma impactar a eficiência nas empresas, reduzindo custos relacionados a desperdícios, redundâncias, bem como inabilidades nos processos 
de tomada de decisão, tornando-se assim um catalisador de organizações economicamente mais saudáveis. Do mesmo modo, fica clara sua relação com o comportamento individual no trabalho (Cunha, Yokomizo e Capellini, 2011; Schein, 2009), o que também traz implicações para a gestão de pessoas nas empresas públicas, uma vez que a cultura dessas empresas pode constituir uma oportunidade ou desafio para promover atitudes mais positivas entre os funcionários e o melhor desempenho de suas funções.

No entanto, este estudo tem algumas limitações que devem ser notadas. Em primeiro lugar, não é possível assumir que as percepções dos participantes das três empresas sejam exatamente as mesmas que as percepções do conjunto total de trabalhadores dessas organizações, ficando as conclusões limitadas aos funcionários da área comercial de empresas do setor industrial. Porém, a decisão por conduzir a pesquisa com funcionários de um único setor, no mesmo nível hierárquico e que têm as mesmas atribuições, foi tomada para aumentar a comparabilidade de percepções e comportamentos nas empresas. Neste estudo, variáveis de controle foram utilizadas para minimizar os efeitos de diferenças individuais demográficas nas amostras. Ainda assim, não é possível atribuir causalidade às relações observadas no estudo, em função de se tratar de um estudo de campo não experimental. Portanto, não é possível descartar a possibilidade de que variações em aprendizagem contínua e diferenças em atitudes e comportamentos observadas sejam covariantes afetadas por outros elementos não investigados. Por fim, apesar de não se poder descartar a presença de efeitos associados ao uso do mesmo método e fonte no levantamento das variáveis atitudinais e comportamentais estudadas, essa limitação não se aplica às análises sobre a influência do tipo de empresa, cuja métrica é objetiva.

Vale ressaltar também que é possível que algumas empresas públicas em outros segmentos que tenham o conhecimento como missão central, como no campo da cultura e da pesquisa, sejam dotadas de culturas mais fortemente marcadas pela aprendizagem contínua. Por outro lado, é também possível que empresas privadas em alguns segmentos não apresentem a aprendizagem contínua como um traço dominante de sua cultura. Assim, é recomendável que estudos semelhantes sejam realizados em outros segmentos, uma vez que esta pesquisa se limitou a investigar apenas funcionários da área comercial de empresas competidoras em um único setor.

Sugere-se também que mais estudos qualitativos sejam realizados sobre o tema, como forma de buscar uma compreensão mais sistemática das barreiras e facilitadores da implantação de uma cultura de aprendizagem contínua nas empresas, a fim de abrir caminho também para desenvolvimento de um know-how na implantação efetiva desse enfoque nas organizações e de seu estímulo. Outra vertente seria a ampliação de investigações quantitativas sobre as associações entre a cultura de aprendizagem contínua e resultados no nível organizacional, tanto resultados financeiros como relativos à satisfação de clientes nas empresas de serviços.

Em suma, parece fundamental que gestores se preocupem com o desenvolvimento de um ambiente que facilite comportamentos ligados à promoção do conhecimento e da aprendizagem nas organizações. Ao fomentar tal cultura, esses gestores certamente estarão plan- 
tando as sementes para cultivar o capital humano de suas empresas, e para se diferenciar como organizações eficientes e que agregam valor em suas áreas de atuação. No entanto, este tende a ser um desafio considerável, particularmente para empresas públicas brasileiras, nas quais a aprendizagem contínua estaria tipicamente menos presente, o que sugerem os resultados deste estudo. No entanto, maior atenção a essa questão teria o potencial de estimular a capacidade de inovação e a qualidade em quaisquer empresas, e notadamente de promover atitudes mais positivas e uma atuação mais eficaz entre seus empregados. Assim, seja para o bem dos acionistas, seja para o bem comum, a valorização e o foco na aprendizagem contínua parecem ser um caminho profícuo e recomendável para todas as organizações.

\section{Referências}

ABBAD, Gardênia et al. Medidas de suporte em avaliação de TD\&E. In: BORGES-ANDRADE, Jairo E.; ABBAD, Gardênia S.; MOURÃO, Luciana (Org.). Treinamento, desenvolvimento e educação em organizações e trabalho: fundamentos para a gestão de pessoas. Porto Alegre: Artmed, 2006. p. 395-422.

ALAVI, Maryam; LEIDNER, Dorothy E. Knowledge management systems: issues, challenges, and benefits. Communications of the AIS, v. 1, n. 2, p. 1, 1999.

ALAVI, Maryam et al. An empirical examination of the influence of organizational culture on knowledge management practices. Journal of Management Information Systems, v. 22, n. 3, p. 191-224, 2006.

ANGELONI, Maria T.; GROTTO, Daniela. A influência na cultura organizacional no compartilhamento do conhecimento. Revista de Administração Faces Journal, v. 8, n. 2, p. 76-93, 2009.

ATINC, Guclu et al. Control variable use and reporting in macro and micro management research. Organizational Research Methods, v. 15, n. 1, p. 57-74, 2011.

AVELAR, Ewerton A. et al. Gestão do conhecimento: uma análise das pesquisas brasileiras desenvolvidas na primeira década do século XXI. Perspectivas em Gestão \& Conhecimento, João Pessoa, v. 1, n. 2, p. 150-165, 2011.

BARRETO, Leilianne M. T. S. et al. Cultura organizacional e liderança: uma relação possível? Revista de Administração, v. 48, n. 1, p. 34-52, 2013.

BASTOS, Antonio V. B.; SIQUEIRA, Mirlene M. M.; GOMES, Ana C. P. Cidadania organizacional. In: SIQUEIRA, Mirlene M. M. (Org.). Novas medidas do comportamento organizacional: ferramentas de diagnóstico e gestão. Porto Alegre: Artmed, 2014. p. 79-103.

BASTOS, Antônio et al. Conceito e perspectivas de estudo das organizações. In: ZANELLI, José C.; BORGES-ANDRADE, Jairo E.; BASTOS, Antônio V. B. (Org.). Psicologia, organizações e trabalho no Brasil. Porto Alegre: Artmed, 2004. p. 63-90.

BATESON, Gregory. Steps to an ecology of mind. Nova York: Ballantine Books, 1972. 
BENDASSOLLI, Pedro F.; MALVEZZI, Sigmar. Desempenho no trabalho: definições, modelos teóricos e desafios à gestão. In: BORGES, Lívia O.; MOURÃO, Luciana. O trabalho e as organizações: atuações a partir da psicologia. Porto Alegre: Artmed, 2013. p. 53-86.

BIANCHI, Márcia et al. A responsabilidade social como parte integrante da cultura organizacional em empresas socialmente responsáveis: análise de conteúdo entre a prática e o discurso. Revista Eletrônica de Estratégia \& Negócios, v. 6, n. 1, p. 160-191, 2013.

BORMAN, Walter C.; MOTOWIDLO, Stephan J. Expanding the criterion domain to include elements of contextual performance. In: SCHMITT, Neal; BORMAN, Walter C. (Ed.). Personnel selection in organizations. San Francisco: Jossey-Bass, 1993. p. 71-98.

BRITO, Lydia M. P. et al. Gestão do conhecimento numa instituição pública de assistência técnica e extensão rural do Nordeste do Brasil. Rev. Adm. Pública, Rio de Janeiro, v. 46, n. 5, p. 341-366, 2012.

CAMPBELL, John P. et al. A theory of performance. In: SCHMITT, Neal; BORMAN, Walter C. Personnel selection in organizations. San Francisco, CA: Jossey-Bass, 1993. p. 35-69.

CAMPOS, Luiz F. B. Análise da nova gestão do conhecimento: perspectivas para abordagens críticas. Perspectivas em Ciência da Informação, v. 12, n. 1, p. 104-122, 2007.

CARDOSO, Olinda N. P.; MACHADO, Rosa T. M. Gestão do conhecimento usando data mining: estudo de caso na Universidade Federal de Lavras. Rev. Adm. Pública, Rio de Janeiro, v. 42, n. 3, p. 495-528, 2008.

CHIN, Wynne W. How to write up and report PLS analyses. In: VINZI, Vincenzo et al. (Ed.). Handbook of partial least squares: concepts, methods and applications. Heidelberg: Springer-Verlag, 2010. p. 655-690.

CUNHA, Julio A. C. et al. Gestão do conhecimento em transnacionais: o ambiente organizacional como instrumento disseminador. Revista de Gestão da Tecnologia e Sistemas de Informação, v. 8, n. 1, p. 213-236, 2011.

CUYPER, Nele de et al. Employability and employees' well-being: mediation by job insecurity. Applied Psychology: An International Review, v. 57, n. 3, p. 488-509, 2008.

DUBIN, Samuel S. Maintaining competence through updating. In: WILLIS, Sherry L.; DUBIN, Samuel S. (Ed.). Maintaining professional competence. San Francisco: Jossey-Bass, 1990. p. 9-43.

DUTRA, Joel S. (Org.). Gestão de carreiras na empresa contemporânea. São Paulo: Atlas, 2010.

ECONOMIST INTELLIGENCE UNIT. Global firms in 2020: the next decade of change for organisations and workers. Londres: Economist Intelligence Unit, 2010.

FARIA, Maria F. B.; FONSECA, Marcus V. A. Medidas de cultura de inovação e de cultura organizacional para análise da associação com inovação. Revista de Administração e Inovação, v. 11, n. 3, p. 30-55, 2014.

GOMIDE JR., Sinésio. Antecedentes e consequentes das percepções de justiça no trabalho. 136 f. Tese (doutorado em psicologia) - Universidade de Brasília, Brasília, 1999. 
HACKMAN, Richard J.; OLDHAM, Greg R. Work redesign. Reading, MA: Addison-Wesley, 1980.

HAIR, Joseph et al. Análise multivariada de dados. 6. ed. Porto Alegre, RS: Bookman Companhia Ed., 2009.

HOFSTEDE, Geert; MCCRAE, Robert R. Personality and culture revisited: linking traits and dimensions of culture. Cross-Cultural Research, v. 38, n. 1, p. 52-88, 2004.

JACKSON, Susan E.; HITT, Michael A.; DENISI, Angelo S. Managing knowledge for sustained competitive advantage: designing strategies for effective human resource management. São Francisco: Jossey-Bass, 2003.

JAWAHAR, Jim I. M. mediating role of satisfaction with growth opportunities on the relationship between employee development opportunities and citizenship behaviors and burnout. Journal of Applied Social Psychology, v. 42, n. 9, p. 2257-2284, 2012.

KENNEDY, Peter W.; KING, Ian P. Economic progress and skill obsolescence with network effects. Economic Theory, v. 26, n. 1, p. 177-201, 2005.

KOTTER, John P.; HESKETT, James L. Corporate culture and performance. Nova York: Free Press, 2011.

KUVAAS, Bard; DYSVIK, Anders. Perceived investment in permanent employee development and social and economic exchange perceptions among temporary employees. Journal of Applied Social Psychology, v. 39, n. 10, p. 2499-2524, 2009.

LEE, Chay H.; BRUVOLD, Norman T. Creating value for employees: investment in employee development. International Journal of Human Resource Management, v. 14, n. 6, p. 981-1000, 2003.

MARKUS, Lynne M. Toward a theory of knowledge reuse: types of knowledge reuse situations and factors in reuse success. Journal of Management Information Systems, v. 18, n. 1, p. 57-93, 2001.

MASSA, Silvia; TESTA, Stefania. A knowledge management approach to organizational competitive advantage: Evidence from the food sector. European Management Journal, v. 27, n. 2, p. 129-141, 2009.

MILLER, Danny A. A preliminary typology of organizational learning: synthesizing the literature. Strategic Management Journal, v. 22, n. 3, p. 484-505, 1996.

MORAES, Lúcio F. R.; KILIMNIK, Zélia M. A qualidade de vida no trabalho burocrático automatizado. Belo Horizonte: UFMG, 1989. (Relatório de Pesquisa).

MORAES, Lúcio F. R.; KILIMNIK, Zélia M. Comprometimento organizacional, qualidade de vida e stress no trabalho: uma abordagem de diagnóstico comparativo. Belo Horizonte: Cepead/Face/ UFMG, 1994.

MORENO, Valter de A.; OLIVEIRA JR., Raul S. de. Avaliação da intenção de uso efetivo de sistemas ERP após a sua estabilização: uma extensão do modelo TAM. Revista Latinoamericana y del Caribe de la Asociación de Sistemas de Información (Relcasi), v. 3, n. 1, p. 9-29, 2010. 
MORSCHEL, Emilio L. et al. A influência da cultura organizacional no processo de inovação: o caso da Águia sistemas de armazenagem em Ponta Grossa, Paraná. Revista de Administração e Inovação, v. 10, n. 2, p. 219-237, 2013.

NONAKA, Ikujiro. A dynamic theory of organizational knowledge creation. Organization Science, v. 5, n. 1, p. 14-37, 1994.

NONAKA, Ikujiro; TAKEUCHI, Hirotaka. Criação do conhecimento na empresa: como as empresas japonesas geram a dinâmica da inovação. Rio de Janeiro: Campus, 1997.

ORGAN, Denis W.; PODSAKOFF, Phillip M.; MACKENZIE, Scott B. Organizational citizenship its nature, antecedents, and consequences. Thousands Oaks, CA: Sage Publications, 2006.

PANTOJA, Maria J.; FREITAS, Isa A. Desenvolvimento e validação da escala de suporte à aprendizagem contínua. In: CONGRESSO NORTE-NORDESTE DE PSICOLOGIA, III, João Pessoa. Anais... 2003.

PEIXOTO, Adriano L. A.; CAETANO, Antônio. Avaliação de desempenho. In: BORGES, Lívia O.; MOURÃO, Luciana (Org.). O trabalho e as organizações: atuações a partir da psicologia. Porto Alegre: Artmed, 2013. p. 528-556.

PIRES, José C. S.; MACEDO, Kátia B. Cultura organizacional em organizações públicas no Brasil. Rev. Adm. Pública, Rio de Janeiro, v. 40, n. 1, p. 81-105, 2006.

PLOYHART, Robert E.; MOLITERNO, Thomas P. Emergence of the human capital resource: a multilevel model. Academy of Management Review, v. 36, n. 1, p. 127-150, 2011.

PODSAKOFF, Nathan P. et al. Individual- and organizational-level consequences of organizational citizenship behaviors: a meta-analysis. Journal of Applied Psychology, v. 94, n. 1, p. 122-141, 2009.

PODSAKOFF, Philip M. et al. Organizational citizenship behaviors: a critical review of the theoretical and empirical literature and suggestions for future research. Journal of Management, v. 26, n. 3, p. 513-563, 2000.

REGO, Arménio; CUNHA, Miguel P. Workplace spirituality and organizational commitment: an empirical study. Journal of Organizational Change Management, v. 21, n. 1, p. 53-75, 2008.

RINGLE, Christian M.; WENDE, Sven; WILL, Alexander. Smart PLS. Disponível em: <www.smartpls. de>. Acesso em: 28 mar. 2014.

ROCHA, Irani et al. A evidenciação da cultura organizacional voltada para a responsabilidade ambiental. Revista Eletrônica de Estratégia \& Negócios, v. 4, n. 1, p. 108-132, 2011.

ROSOW, Jerome M.; ZAGER, Robert. Training: the competitive edge. San Francisco, CA: JosseyBass, 1988.

ROUSSEAU, Denise. Psychological contracts in organizations: understanding written and unwritten agreements. Thousand Oaks, CA: Sage, 1995.

SANTOS, Nathália M. et al. Cultura organizacional, estrutura organizacional e gestão de pessoas como bases para uma gestão orientada por processos e seus impactos no desempenho organizacional. Brazilian Business Review, v. 11, n. 3, p. 106-129, 2014. 
SCHEIN, Edgar H. Cultura organizacional e liderança. São Paulo: Atlas, 2009.

SCHIEMANN, William A. Reinventing talent management: how to maximize performance in the new marketplace. New Jersey, NJ: John Wiley \& Sons, 2009.

SERENKO, Alexander; BONTIS, Nick. Meta-review of knowledge management and intellectual capital literature: citation impact and research productivity rankings. Knowledge and Process Management, v. 11, n. 3, p. 185-198, 2004.

SESSA, Valerie I.; LONDON, Manuel. Continuous learning in organizations: individual, group, and organizational perspectives. Mahwah, NJ: Taylor \& Francis, 2006.

SILVA, Lindomar P.; FADUL, Élvia. A produção científica sobre cultura organizacional em organizações públicas no período de 1997 a 2007: um convite à reflexão. Revista de Administração Contemporânea, v. 14, n. 4, p. 651-669, 2010.

SILVA, Lindomar P.; FADUL, Élvia. Cultura organizacional em organização pública: as bases da mudança organizacional a partir da reforma gerencial. In: SIMPÓSIO DE EXCELÊNCIA EM GESTÃO E TECNOLOGIA, 2007, Resende. Anais... 2007.

SILVA, Narbal; ZANELLI, José C.; TOLFO, Suzana R. Diagnóstico e gestão da cultura nas organizações. In: BORGES, Lívia O.; MOURÃO, Luciana. O trabalho e as organizações: atuações a partir da psicologia. Porto Alegre: Artmed, 2013.

SIQUEIRA, Mirlene M. M. Proposição e análise de um modelo para comportamentos de cidadania organizacional. Revista de Administração Contemporânea — RAC, v. 7, n. especial, p. 165-184, 2003.

SIQUEIRA, Mirlene M. M.; GOMIDE JR., Sinésio. Vínculos do indivíduo com o trabalho e com a organização. In: ZANELLI, José C.; BORGES-ANDRADE, Jairo E.; BASTOS, Antonio V. B. (Org.). Psicologia, organizações e trabalho no Brasil. Porto Alegre: Artmed, 2004. p. 300-330.

SOARES, Daniela P. S. et al. A influência do ambiente e da cultura organizacional no papel da área de recursos humanos: um estudo comparativo de casos em duas empresas de médio porte. Revista Alcance, v. 19, n. 4, p. 441-460, 2012.

SOUZA, Fernando H. et al. Interseção entre a gestão do conhecimento e a cultura organizacional: um estudo sobre a percepção dos gerentes de projetos. Revista de Gestão e Projetos, v. 5, n. 2, p. 51-67, 2014.

SOUZA, Maria T. S. et al. Ética e liderança: sua influência na cultura organizacional da empresa. Revista de Administração e Inovação, v. 1, n. 1, p. 89-100, 2004.

SPECTOR, Paul; BRANNICK, Michael. Methodological urban legends: the misuse of statistical control variables. Organizational Research Methods, v. 14, n. 2, p. 287-305, 2010.

TERRA, José C. C. Gestão do conhecimento: o grande desafio empresarial: uma abordagem baseada no aprendizado e na criatividade. São Paulo: Negócio, 2000.

TRACEY, J. Bruce et al. Applying trained skills on the job: the importance of the work environment. Journal of Applied Psychology, v. 80, n. 2, p. 239-252, 1995. 
URBACH, Nils; AHLEMANN, Frederik. Structural equation modeling in information systems research using partial least squares. Journal of Information Technology Theory and Application, v. 11, n. 2, p. 5-40, 2010.

VASCONCELOS, Isabella; MASCARENHAS, André O.; VASCONCELOS, Flávio C. Paradoxos organizacionais, gestão de pessoas e tecnologia na Souza Cruz. Revista de Administração de Empresas, v. 3, n. 2, p. 1-20, 2004.

WALDMAN, David A.; AVOLIO, Bruce J. Aging and work performance in perspective: contextual and developmental considerations. In: FERRIS, Gerald R.; ROWLAND, Kendrith M. (Ed.). Research in personnel and human resources management. Greenwich, CT: JAI Press, 1993. p. 133-162.

WETZELS, Martin et al. Using PLS path modeling for assessing hierarchical construct models: guidelines and empirical illustration. MIS Quarterly, v. 33, n. 1, p. 177-195, 2009.

ZANELLI, José C.; SILVA, Narbal. Interação humana e gestão: a construção psicossocial das organizações de trabalho. São Paulo: Casa do Psicólogo, 2008.

Flávia de Souza Costa Neves Cavazotte é PhD em administração/comportamento organizacional; professora do IAG Escola de Negócios da Pontifícia Universidade Católica do Rio de Janeiro (PUC-Rio). E-mail: flavia.cavazotte@iag.puc-rio.br.

Valter de Assis Moreno Jr. é PhD em administração; professor adjunto das faculdades Ibmec (RJ). E-mail: vmoreno@ibmecrj.br.

Lucas Martins Turano é MSc em administração pelo IAG da Pontifícia Universidade Católica do Rio de Janeiro (PUC-Rio); administrador no BNDES. E-mail: lucas.turano2@gmail.com. 